\title{
Personal Travelling Scheduler
}

\author{
Abhishek Tapadia ${ }^{1}$, Kunal Agarwal ${ }^{2}$, Brijesh sonawane ${ }^{3}$, Shubham Akurdekar ${ }^{4}$, Prof. Suchita Wankhede ${ }^{5}$ \\ Student, Comp Dept, KJEI'S Trinity College of Engineering and Research, Pune, India ${ }^{1,2,3,4}$ \\ Guide, Comp Dept, KJEI'S Trinity College of Engineering and Research, Pune, India ${ }^{5}$
}

\begin{abstract}
Tour Guide as the name goes is an advance yet highly promising system helping a tourist or any user to get accurate and best data in no time. This System is an Android Application and Uses Android Studio as its Front End and SQL Server as its Back End. The Application acts as a Tour Guide giving out outputs to the user for every input given to the system. The System is highly reliable as it uses google map API which are very accurate and same goes for the weather conditions, personal interest and budget to travel. This System tries the user to gives a heads-up giving the weather conditions to make sure that the user will be comfortable to visit the desired place. The User has options to select for the places he wants to visit for instance parks, beaches monuments or food joints and so on; the system will ask whether he is searching for the current. Locality or some other place.
\end{abstract}

Keywords: TSP, Geo-fencing, Shortest Path, Daily Tourism Behavior

\section{INTRODUCTION}

In this project we propose an integrated methodology for urban mobility, especially touristic mobility, based on innovative approaches regarding urban public transport with the aim to develop sustainable mobility network and implementing integrated and comprehensive policies for sustainable urban planning, related to the environment. The System is changing places and makes use of Google maps to display places if the user wishes to within particular distance mentioned by the user considering a fence of geo locations.

\section{LITERATURE SURVEY}

“ANALOC: Efficient analytics over Location Based Services." md farhadur rahman, saad bin suhaim, weimo liu, saravanan thirumuruganathan, nan zhang, gautam das.

ANALOC, a prototypical system for enabling the analytics of data underlying real-world Location Based Services (LBS) by using nothing but the k-Neares Neighbour (knn) search interface (often web-based) publicly provided by the LBS. Location Based Services (LBS), including standalone ones such as Google Maps and embedded ones such as "users near me" in the we chat instant-messaging platform, provide great utility to Millions of users.

"The new technique based on the galaxy based search algorithm for solving the symmetric travelling salesman problem." Ajchara Phu-ang

The proposed algorithm is based on the concept of the galaxy based search algorithm with the new technique called the clockwise search process. In the first step, introduce new rules to generate the initial solutions. The hill climbing local search is employed to search the new solution in surrounding areas of the initial solutions in the second step. The proposed algorithm use of the clockwise search process to generate more diversity of the new solution. Then, the final step, the hill climbing local search is applied again to increase the local search capabilities.

“A Transformation for Multiple Depot Multiple Traveling Salesman Problem.” Mustafa Assaf, Malick Ndiaye. A transformation for the Multi Depot Multiple TSP (MmTSP) into Asymmetrical TSP (ATSP). The transformation is done by duplicating the depots, and with the aid of a precedence constraint, the salesman in the transformed graph will visit the original depot and its duplicates before proceeding to the next depots. We proved that solving the transformed problem leads to the same optimal solution. We test the proposed transformation against a Mixed Integer Linear Programming formulation for the MmTSP and compare the computational time between the two.

\section{PROPOSE SYSTEM}

In proposed system we make a android application for people to help to reach near park. In system architecture the user can register the application and registration is successful then login the application. Similarly the Admin can register and login the same application. Admin can add the places and manage the user. System can perform the some application like Analysis of user Interest, Suggest place, and show the route on suggestion place. And using database 


\section{International Journal of Advanced Research in Computer and Communication Engineering}

Vol. 7, Issue 11, November 2018

operation this data can be stored in database. The User give the current location and using geo fencing algorithm they can search the near park.

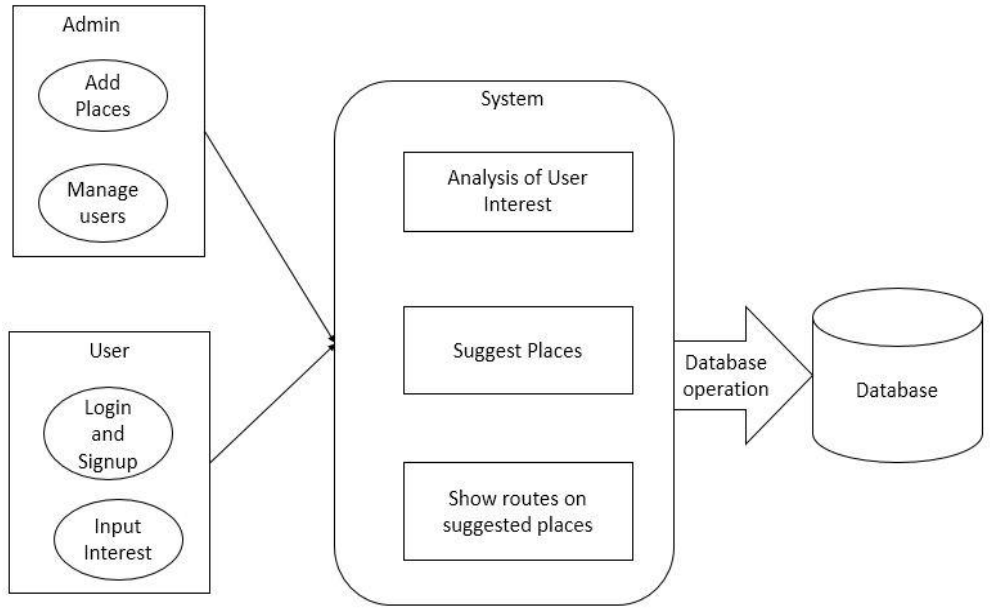

Figure 1: system architecture

In system architecture the user and admin can register android application. Registration is successful he login the application. Admin can add the place and manage the user and this information give to system. Then user give the input interest and give the system. Then system can analysis user interest, Suggestion of places and manage the route this type of operation system can be perform save save it to database.

\section{METHODOLOGIES}

In that some method are used like GPS. Using GPS they can find the location. Global Positioning System (GPS) wherever you are on the planet, at least four GPS satellites are 'visible' at any time. Each one transmits information about its position and the current time at regular intervals. These signals, travelling at the speed of light, are intercepted by your GPS receiver . using Google map they can display the route.

\section{APPLICATION}

1. This application can be used for analysis of user based on visited place.

2. We can improve the count of visitors with good review based system. Considering budget of user we can suggest them a good plan to save money and time

\section{CONCLUSION}

For user or tourism they don't know about the near park so using this application they can find the near park and reach to destination as early as possible.

\section{REFERRENCES}

[1]. W.Lizhe, Jie Tao, M.Kunze, A.C. Castellanos, D.Kramer, and W.Karl,"Scientific Cloud Computing: Early Definition and Experience," In HPCC, vol. 8, pp. 825-830. Sep 2008.

[2]. P.K.Paul and M.K.Ghose, "Cloud computing: possibilities, challenges and opportunities with special reference to its emerging need in the academic and working area of Information Science," In Procedia Engineering, vol. (23), pp.2222-2227, Jan 2012.

[3]. Kandukuri, Balachandra Reddy, and Atanu Rakshit, "Cloud security issues," InServices Computing, 2009. SCC\&\#39;09. IEEE International Conference on IEEE, pp. 517-520, Sep 2009.

[4]. L. Adleman, "Molecular computation of solutions to combinational problems".American Association for the Advancement of Science, pp.1021-1024, 1994.

[5]. Rachna.A, and Anshu.P, "Maintaining Data Confidentiality and Security over Cloud: An Overview", International Journal of Engineering Research and Applications (IJERA), Vol. (4), pp.1922-1926, July 2013.

[6]. D.Sureshraj and Dr.V.Murali Bhaskaran, Automatic DNA Sequence Generation for Secured Effective Multi-Cloud Storage, Journal of Computer Engineering (IOSR-JCE), vol.15, pp. 86-94, Nov-Dec 20 